\title{
An Update for Mesenchymal Stem Cell Therapy in Lupus Nephritis
}

\author{
Wenchao Li Weiwei Chen Lingyun Sun \\ Department of Rheumatology and Immunology, The Affiliated Drum Tower Hospital of Nanjing University Medical \\ School, Nanjing, China
}

\section{Keywords}

Mesenchymal stem cells · Lupus nephritis · Stem cell

disorder · Dysfunction · Immunoregulation

\begin{abstract}
Background: Lupus nephritis (LN) is the most severe organ manifestations of systemic lupus erythematosus (SLE). Although increased knowledge of the disease pathogenesis has improved treatment options, outcomes have plateaued as current immunosuppressive therapies have failed to prevent disease relapse in more than half of treated patients. Thus, there is still an urgent need for novel therapy. Mesenchymal stem cells (MSCs) possess a potently immunosuppressive regulation on immune responses, and intravenous transplantation of MSCs ameliorates disease symptoms and has emerged as a potential beneficial therapy for LN. The objective of this review is to discuss the defective functions of MSCs in LN patients and the application of MSCs in the treatment of both LN animal models and patients. Summary: Bone marrow MSCs from SLE patients exhibit impaired capabilities of migration, differentiation, and immune regulation and display senescent phenotype. Allogeneic MSCs suppress autoimmunity and restore renal function in mouse
\end{abstract}

(C) 2021 The Author(s)

Published by S. Karger AG, Basel

www.karger.com/kdd

Karger ${ }^{\prime \prime} \div$

BOPEN ACCESS
This article is licensed under the Creative Commons AttributionNonCommercial-NoDerivatives 4.0 International License (CC BYNC-ND) (http://www.karger.com/Services/OpenAccessLicense) Usage and distribution for commercial purposes as well as any distribution of modified material requires written permission. models and patients with LN by inducing regulatory immune cells and suppressing Th1, Th17, T follicular helper cell, and B-cell responses. In addition, MSCs can home to the kidney and integrate into tubular cells and differentiate into mesangial cells. Key Messages: The efficacy of MSCs in the LN treatment remains to be confirmed, and future advances from stem cell science can be expected to pinpoint significant MSC subpopulations, as well as specific mechanisms of action, leading the way to the use of more potent stimulated or primed pretreated MSCs to treat LN.

(c) 2021 The Author(s) Published by S. Karger AG, Basel

\section{Introduction}

Systemic lupus erythematosus (SLE) is a life-threatening autoimmune inflammatory disease involving a variety of autoantibodies, which are produced by overactivated B cells and circulate in peripheral blood or deposit in organs [1]. Deposition of autoantibodies triggers the formation of immune complexes and then leads to tissue

Wenchao Li and Weiwei Chen contributed equally to this article.

Lingyun Sun

Department of Rheumatology and Immunology

The Affiliated Drum Tower Hospital of Nanjing University Medical School

321 Zhongshan Road, Nanjing 210008 (China)

lingyunsun@nju.edu.cn 
inflammation in multiple organs, including blood vessels, joints, kidneys, and skin [2]. Renal involvement, termed lupus nephritis (LN), occurs in approximately $50-60 \%$ of the patients and remains one of the most serious visceral complications in SLE [3, 4]. Men with SLE tend to have more aggressive disease with higher rates of renal and cardiovascular involvements and are more likely to develop kidney failure than women [5]. According to the statistics of the Chinese SLE Treatment and Research group (CSTAR), LN causes the highest standardized mortality ratio in China [6].

Clinically, LN is characterized by proteinuria, cellular casts, hematuria, and even renal failure, which may lead to end-stage renal disease and the need for peritoneal dialysis, hemodialysis, or renal transplantation. After decades of research, several drugs are available now, including corticosteroids, hydroxychloroquine, cyclophosphamide (CTX), methotrexate, tacrolimus, azathioprine, and mycophenolate mofetil (MMF) and novel biological agents (rituximab and belimumab antibody) [7]. Currently, corticosteroids combined with either CTX or MMF have been established as the standardized care of LN patients [8]. However, the largest controlled trial comparing MMF with intravenous CTX in LN achieved complete remission (serum creatinine, urine protein, and urine sediment were all met) in only 8.6 and $8.1 \%$ of patients, respectively [9]. An analysis of LN patients even showed that there had not been a major improvement in outcome in the last 30 years to 2011 [10], indicating that the conventional drugs are unlikely to produce any further clinically important improvements. Aside from the poor efficacy, long-term usage of nonspecific immunosuppressive regimens may increase the risk of serious infection and secondary malignant tumors; besides, because of its high cost, the use of biological agents is also limited $[11,12]$. Thus, there is an urgent need for safer and more effective treatments for LN. In recent years, mesenchymal stem cell (MSC) transplantation has been used to treat autoimmune diseases and benefits patients that are resistant to the conventional therapies. Our 6-year follow-up observational study has provided evidence for the safety and efficacy of allogeneic umbilical cord-derived MSCs (UC-MSCs) in treating refractory SLE patients [13].

MSCs were firstly described as bone-forming cells, and they were reported to display adult stem cell multipotency [14]. Because MSCs lack specific molecular markers, in 2006, the International Society for Cellular Therapy (ISCT) recommended minimal criteria to identify MSCs. (1) Fibroblast like and must be plastic adherent under standard culture conditions. (2) Express CD90, CD73, and CD105 and lack expression of CD45, CD34, CD14 (or CD11b), CD79A (or CD19), and HLA-DR surface molecules. (3) Differentiate into osteoblasts, adipocytes, and chondrocytes under standard in vitro differentiating conditions [15]. While the pluripotential properties initially put MSCs in the center stage of an alleged era of regenerative medicine, their unique immunoregulatory property makes them an attractive choice for treating autoimmune diseases.

MSCs exert their immunoregulatory effects by secreting soluble factors or directly interacting with a variety of immune effector cells, including both innate and adaptive immune cells. They can either educate macrophages, monocytes, and dendritic cells (DCs) to an anti-inflammatory phenotype or suppress proliferation of CD4 and CD8 $\mathrm{T}$ lymphocytes in vitro in a dose-dependent, nonapoptotic-induced manner or promote the proliferation and differentiation of regulatory $\mathrm{T}$ cells (Treg). Now, it has been well known that transforming growth factor- $\beta$ (TGF- $\beta$ ), prostaglandin E2 (PGE2), nitric oxide (NO), programmed cell death 1 ligand 1 (PD-L1), and indoleamine 2,3-dioxygenase (IDO) are involved in the MSCmediated immunosuppression. Recently, it has been reported that MSCs can also exert immunoregulatory effects by release of microvesicles, which may interact with target cells by surface-expressed ligands, transfer surface receptors, deliver proteins, mRNAs, and bioactive lipids.

In clinical trials, the efficacy of MSCs in treating LN is still controversial. The reason might be that there were many confounding factors among the patients, such as various disease severity and different treatment regimens. In this review, the defective function of MSCs in LN patients is discussed, followed by a summary of the application of MSCs in the treatment of both LN animal models and patients.

\section{MSC Dysfunction in SLE}

Morton and Siegel first reported that autoimmune potential can be transplanted from autoimmune New Zealand Black mice into non-autoimmune strains ( $\mathrm{H}-2 \mathrm{~d}$ histocompatible $\mathrm{DBA} / 2$ and $\mathrm{BALB} / \mathrm{c}$ ). Later on, Ikehara et al. observed that transplantation of normal bone marrow from $\mathrm{C} 3 \mathrm{H} / \mathrm{HeN} \mathrm{nu} / \mathrm{nu}\left(H-2^{k}\right)$ mice prevented thymic abnormalities of young MRL/MP-lpr/lpr (MRL/l; $\left.H-2^{k}\right)$ mice, confirming that defects in stem cells could ultimately lead to the dysfunctions of immune cells that derived and differentiated from them [73]. Gene expression
Li/Chen/Sun 


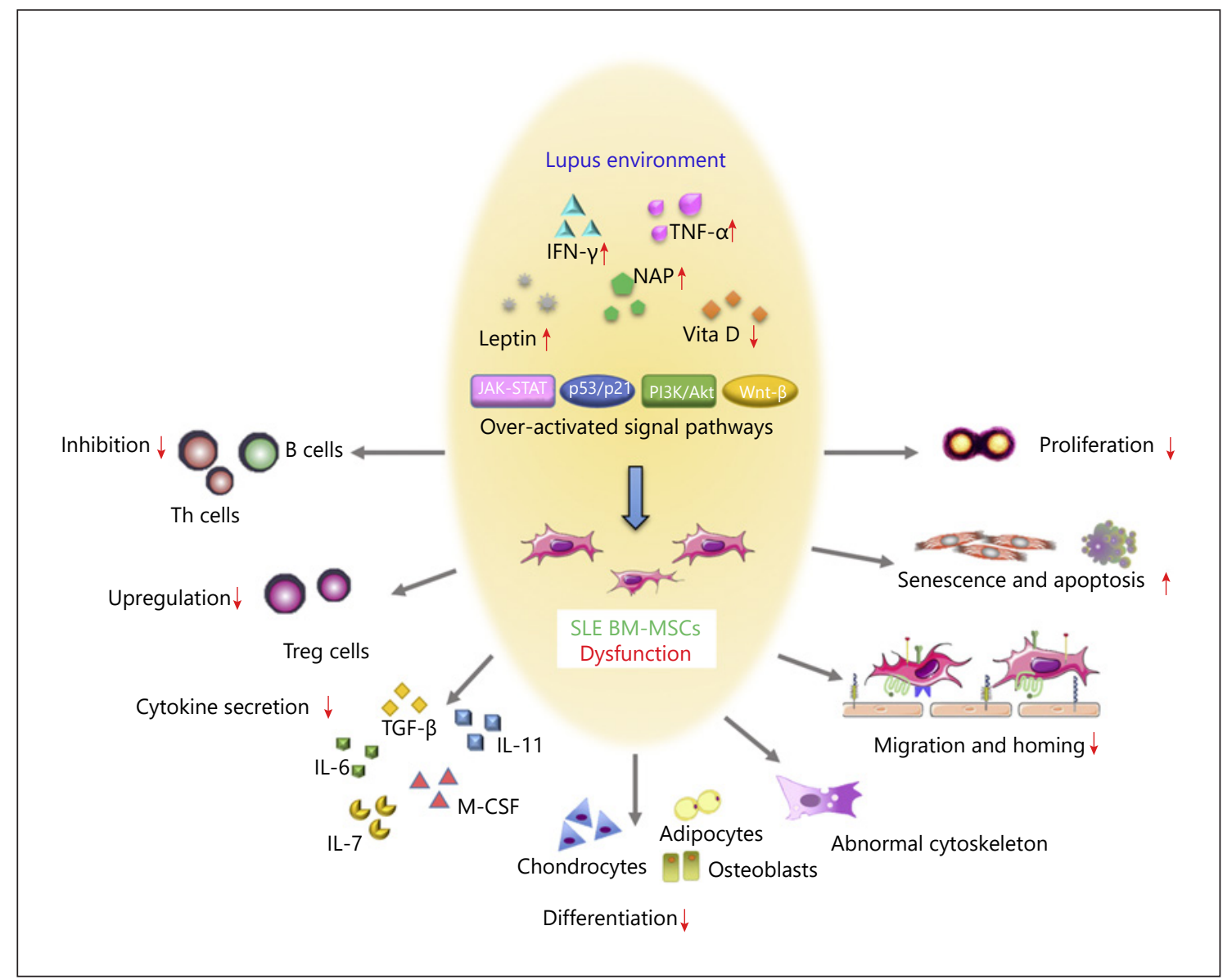

Fig. 1. Dysfunction of BM-MSCs in SLE. BM-MSCs from either lupus mice or patients exhibited impaired capabilities for migration, proliferation, differentiation, and immune modulation and increased senescence. The abnormal in vivo inflammatory environment and several signaling pathways including JAK-STAT, p53/p21, PI3K/ Akt, and Wnt/beta-catenin may be related to the deficiencies of BM-MSCs in SLE. BM-MSCs, bone marrowderived mesenchymal stem cells; SLE, systemic lupus erythematosus; Th cells, T helper cells; NAP, neutrophilactivating protein; Vita D, vitamin D.

profiles of bone marrow-derived MSCs (BM-MSCs) from SLE patients and healthy controls displayed a great difference, in which 652 genes were upregulated and 1,253 were downregulated, confirming that SLE MSCs are quite different from those from healthy controls [16]. In this way, a group of researchers defined SLE as a "stem cell disorder" [17]. MSC dysfunction in SLE is summarized in Figure 1.

Based on this theory, our group proposed that BMMSCs from SLE patients may have certain functional deficiency, which then could be involved in the pathogenesis of the disease. Our data showed that in comparison with healthy controls, SLE patients had much more apoptotic MSCs in the bone marrow. Moreover, BM-MSCs from SLE patients grew slower than those isolated from healthy controls. After 5 passages, SLE BM-MSCs became senile and exhibited old dots, disordered cytoskeletal organization, and increased SA- $\beta$-gal staining, while aging signals began to appear in healthy MSCs after passage 10, suggesting that SLE BM-MSCs age more easily and lose vitality gradually during in vitro culture. To elucidate the underlying mechanism, we checked the molecules involved in aging and apoptosis signal pathways. The data showed that the expression of Bcl-2, which is important in inhibiting apoptosis, was markedly reduced in SLE patient MSCs. When we induced apoptosis of MSCs by tumor necrosis factor- $\alpha$ (TNF- $\alpha$ ) stimulation in vitro, Bax and caspase 8 expression in SLE MSCs increased significantly. More cytochrome-C-positive pellets were detected in the cytosolic fraction of SLE MSCs than in healthy 
controls. Fas and TNF- $\alpha$ receptor 1 in SLE MSCs were significantly upregulated compared with normal controls, and the serum levels of FasL and TNF-a were also elevated, which may be responsible for the increased apoptosis of MSCs [18]. In addition, intracellular reactive oxygen species levels of SLE MSCs were higher than those of normal controls [19]. Endoplasmic reticulum (ER) is an intracellular organelle which performs essential functions in the cell, including protein synthesis, posttranslational modification, and protein folding. ER stress (ERS) response occurs when chaperone proteins perceive and respond to abnormalities of ER function, such as protein folding [20]. If the conditions that trigger ERS are not corrected in time, ERS will induce apoptosis and autophagy of the cell. A study by Gu et al. [21] suggested that the increased ERS was responsible for the distorted, dilated, and swollen morphology of BM-MSCs from SLE patients. Several signaling pathways including JAK-STAT, p53/ p21, PTEN/Akt, PI3K/Akt, and Wnt/beta-catenin were abnormally activated in the development of the senescence phenotype in MSCs from SLE patients [22-25]. Inhibition of these pathways could reverse the senescent phenotype. Mitochondrial antiviral signaling protein (MAVS) is the only adaptor protein between nuclear factor (NF)- $\kappa \mathrm{B}$ and retinoic acid-inducible gene I-like receptors and is downstream of IRF-3/7 signaling pathways [26]. It plays an indispensable role in the innate immune response and induction of interferon (IFN) expression, especially IFN- $\beta$, which can trigger DNA signaling pathways by inducing chemical modification of p53 and induce cellular aging and senescence. MAVS was significantly increased in SLE MSCs, suggesting that MAVS may be involved in the senescence of MSCs. Indeed, silencing of MAVS could downregulate IFN- $\beta$, p53, and p16 proteins and alter cytokine production in SLE MSCs [27].

Except for increased senescence and apoptosis, BMMSCs from SLE patients exhibit impaired capabilities for migration, proliferation, differentiation, and immune modulation $[19,28]$. The impaired osteoblastic differentiation of SLE BM-MSCs may participate in the pathology of osteoporosis in SLE patients [29]. Our group observed decreased mineralization and osteogenic gene expression and impairment of adipogenic differentiation in SLE BMMSCs, displaying impaired osteogenic differentiation. In consistent with our observation in the mouse model, the osteoblastic differentiation ability of BM-MSCs from SLE patients was also lower. Overactivated NF- $\kappa B$ signaling pathway has been shown to be involved in the underlying mechanism. Activated NF- $\kappa$ B pathway in SLE BM-MSCs inhibited bone morphogenic protein-2 (BMP-2)-induced osteoblastic differentiation through the BMP/Smad signaling pathway, and the effects were partially reversed by adding an NF- $\kappa$ B inhibitor, pyrrolidine dithiocarbamate. Moreover, our recent findings suggested that SLE patients displayed a unique miRNA signature in BM-MSCs, among which miR-663 was closely associated with SLE disease activity. We found that miR-663 inhibited the proliferation and migration of BM-MSCs. By targeting TGF- $\beta 1$, miR-663 impaired BM-MSC-mediated downregulation of follicular T helper (Tfh) cells and upregulation of Treg cells. Moreover, overexpression of miR-663 weakened the therapeutic effects of BM-MSCs, while inhibiting miR-663 improved the remission of lupus disease in MRL/lpr mice.

By secreting cytokines, MSCs provide suitable environment for maturation, differentiation, proliferation, and hematopoiesis of hematopoietic stem cells. We found that MSCs of both healthy controls and SLE patients expressed cytokines such as IL-6, IL-7, IL-11, TGF- $\beta$, MCSF, and stromal cell-derived factor. However, the IL-6 and IL-7 mRNA expression was obviously downregulated in MSCs from SLE patients. Interestingly, IL-7 expressed by MSCs was negatively correlated with the SLE disease activity index (SLEDAI) in the SLE patients, and IL-6 reduction restrained MSC-induced macrophage polarization [7]. Most importantly, production of IDO, which is critical for immunoregulatory function of MSCs, was defective in SLE BM-MSCs and led to insufficiency to inhibit T-cell and B-cell proliferation as well as plasma cell terminal differentiation [30].

\section{The Application of MSCs in the Treatment of LN}

\section{Preclinical Studies}

Murine models have greatly contributed to our understanding of the pathogenesis of SLE. Generally, SLE mouse models can be divided into spontaneous and induced models. The most widely used spontaneous lupus mice are the F1 hybrid between the New Zealand Black (NZB) and New Zealand White (NZW) strains (NZB/W F1) and its derivatives and the MRL/lpr strains. The most commonly used lupus model is the pristane-induced lupus model [31].

Both MRL/lpr and NZB/W F1 mice demonstrate progressive nephritis, elevated serum autoantibodies, and immune abnormalities. The efficacy comparison of BMMSC transplantation and CTX in SLE treatment has been done in these 2 models. In MRL/lpr mice, MSC injection 
Table 1. Clinical trials of MSC treatment [39-45]

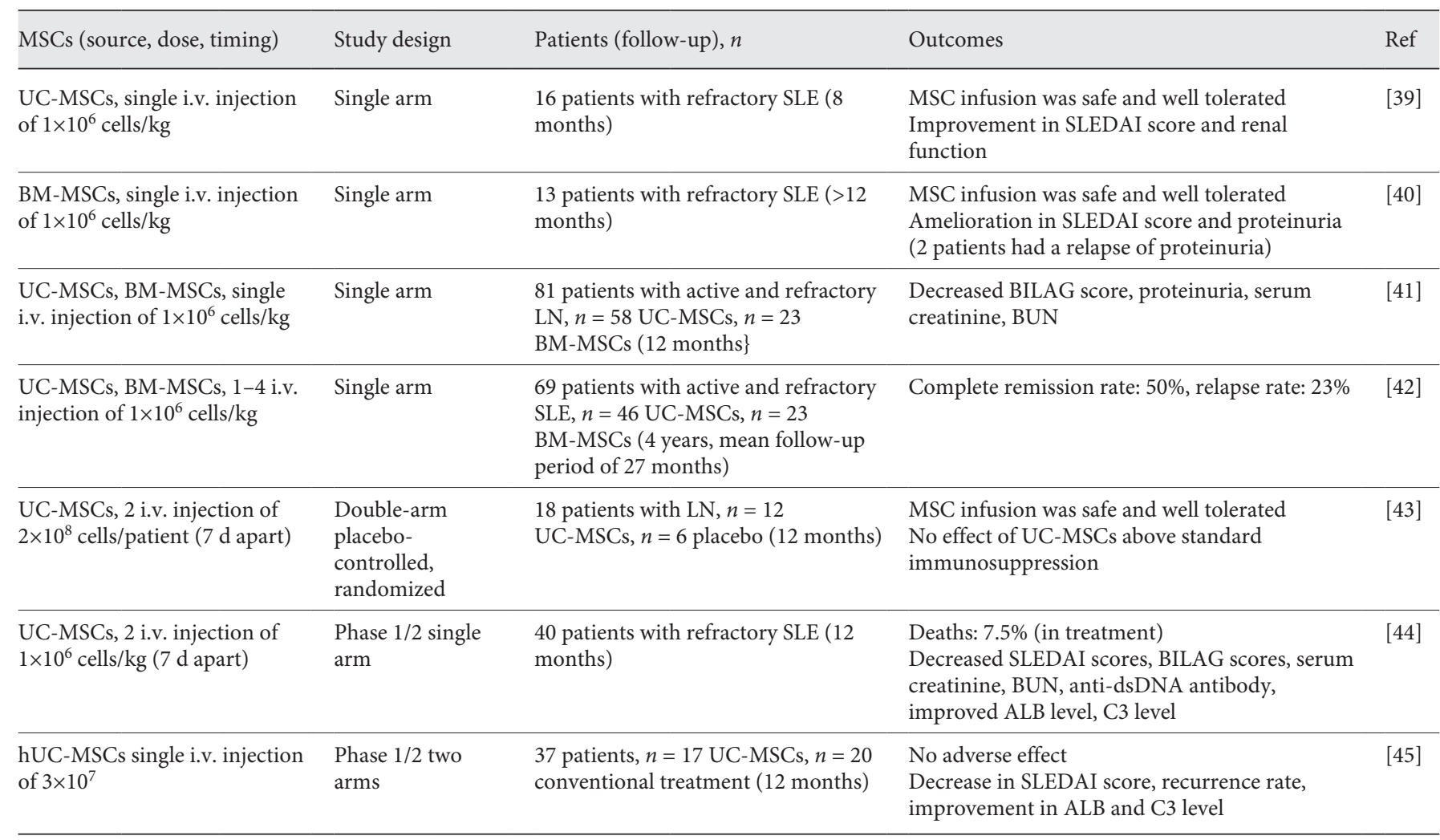

MSC, mesenchymal stem cell; SLE, systemic lupus erythematosus; LN, lupus nephritis; UC-MSCs, umbilical cord-derived MSCs; BM-MSCs, bone marrow-derived MSCs.

significantly reduced serum anti-double-stranded DNA (anti-dsDNA) antibodies and immunoglobulins IgG1, IgG2a, IgG2b, and IgM as well as serum albumin. However, CTX treatment only partially reduced the levels of serum autoantibodies and immunoglobulin IgG2a, restored albumin level, and failed to reduce circulating immunoglobulins IgG1, IgG2b, and IgM. Notably, MSCs improved renal disorders, specifically restoring kidney glomerular structure and reducing $\mathrm{C} 3$ and glomerular IgG deposition. CTX could reduce glomerular IgG deposition, but failed to restore the glomerular structure and C3 accumulation. Interestingly, MSC treatment rather than CTX was able to completely restore renal function, shown as normalization of serum and urine creatinine levels [32]. Furthermore, a study showed that human UCMSC treatment alleviated LN in a nondose-dependent manner. Both single and multiple treatments with UCMSCs could decrease the levels of 24-h proteinuria, serum creatinine, and anti-dsDNA antibodies [33].

Employing the other lupus model of NZB/W F1 mice, Chang et al. [34] showed that treatment with human UC-
MSCs significantly delayed the development of proteinuria, alleviated renal injury, and prolonged survival. Choi et al. [35] found that long-term serial administration of human adipose tissue-derived MSCs ameliorated lupus symptoms without adverse effects. Mice in the MSC-treated group had dramatically higher survival rates with improved histologic and serologic abnormalities as well as decreased incidence of proteinuria [35]. In a similar study, Jang et al. [36] found that infusion of human BM-MSCs attenuated glomerulonephritis. MSCs also decreased levels of autoantibodies and the incidence of proteinuria and increased survival. Infiltration of long-lived plasma cells into the inflamed kidney was significantly reduced in the BM-MSC-treated mice. Importantly, BM-MSCs directly suppressed the in vitro differentiation of naïve $\mathrm{CD} 4 \mathrm{~T}$ cells toward Tfh cells in a contactdependent manner, suggesting that MSCs attenuate LN by suppressing the development of Tfh cells and the subsequent activation of humoral immune components [36]. All these data reveal that different sources of allogeneic MSCs are able to ameliorate disease manifestations and inhibit disease progression in different lupus models. 


\section{Clinical Studies}

The first phase I trial of MSC therapy was carried out by Lazarus et al. [37], demonstrating the safety of ex vivo culture expansion and intravenous infusion of autologous BM-MSCs in 15 patient volunteers. From then on, MSC therapy for immune disorders has been steadily reported as a novel tool to prevent or treat graft-versus-host disease after hematopoietic stem cell transplantation [38]. Based on the results of the encouraging data collected form those preclinical studies of MSCs in treating lupus prone mice, MSC populations have been tested in clinical trials in patients with LN (Table 1).

Our group firstly employed allogeneic BM-MSCs to treat refractory and severe lupus patients in 2007 [39]. In this pilot and preliminary study, 4 patients were chosen, who were treated with CTX and prednisone for $>6$ months, but SLEDAI remained $>8$ and were diagnosed LN without end-stage renal failure. The data showed a stable 12- to 18 -month disease remission in all patients. These patients showed an amelioration of disease activity and improvement in serologic markers and renal functions, suggesting that allogeneic BM-MSC transplantation is a new hope for treatment in refractory LN patients. Therefore, according to this clinical study, we believe that BM-MSC therapy will be a feasible and safe salvage therapy in patients with SLE resistant to conventional treatments, even for those with LN. All of the aforementioned studies used BM-MSCs from patients' relatives or volunteers; however, aspiration of BM-MSCs is invasive, and the quantity of the cells is limited and will take quite a long time to get sufficient expansion of the original cells. Thus, finding a new and available source of MSCs is desired for MSC therapy.

MSCs can be isolated from various tissues, including the umbilical cord, which is a rich source and belongs to medical wastes. In addition, compared with BM nucleated cells, colony-forming unit fibroblast frequency is higher in UC nucleated cells. UC-MSCs have a higher proliferation capacity, indicating that UC-MSCs may be a perfect alternative source for clinical application [46]. In our studies, we enrolled more patients to further determine the safety and efficacy of allogeneic UC-MSC transplantation [39, 41]. All MSC transplantation was conducted in severe and drug-resistant lupus patients. The patients were followed for $>12$ months, exhibiting significant reductions of 24-h proteinuria and improvement of SLEDAI and British Isles Lupus Assessment Group (BILAG) scores. The Cox regression analysis demonstrated significant correlations between complete renal remission and baseline clinical parameters, and renal flare was more likely to occur in patients with higher baseline serum creatinine levels. Notably, no serious adverse events were reported. A further phase II study, with up to 4 years of follow-up (mean 27 months), demonstrated a good clinical safety profile, with an overall rate of survival of $94 \%$, and about $50 \%$ patients achieving and remaining in clinical remission at a 4 -year visit [42]. These studies also showed no difference in clinical efficacy between allogeneic BM-MSC and UC-MSC treatment.

However, in a randomized double-blind, placebo-controlled trial of allogeneic UC-MSC for newly diagnosed severe LN, Deng et al. [43] concluded that the MSCs had no apparent additional effect over and above standard immunosuppression, as they observed that a similar proportion of patients on UC-MSC and placebo achieved complete remission. Improvements in serum albumin, complement, renal function, SLEDAI, and BILAG scores were similar in both groups. Although UC-MSC did not improve the outcomes of standard immunosuppressive treatment, it does not mean they have no immunosuppressive effects at all. In the short term, LN generally responds well to CTX treatment, particularly in Asian patients. Thus, in this situation, there is not much to gain from MSC treatment; therefore, the finding of no additional benefit of MSC therapy was unsurprising.

Over the past few decades, thousands of patients have been included in the clinical studies of MSC therapy, but the size of the study populations has often been relatively small, which limits their scientific value. Given this, our group is recently preparing to conduct large randomized and multicenter studies, which will be sufficiently powered to measure relevant clinical end points and provide mechanistic insights.

\section{The Mechanism of Allogeneic MSC Therapy for LN}

Despite the in vitro and in vivo evidence for the therapeutic effect of MSCs in LN treatment, there are still major questions concerning the optimal dosage, routes of administration, and the fate of the cells after infusion. Thus, it is critical to explore the mechanisms by which MSCs exert their immunomodulatory and reparative effects. Until now, no uniform mechanism has been discovered, but the available data have revealed multiple working models for the beneficial effects of MSCs. According to the current understanding, we summarize the key mechanisms in Figure 2.

\section{MSCs Homing to Kidney}

Homing ability is an important characteristic of MSCs. Accumulating evidence indicated that after administra- 


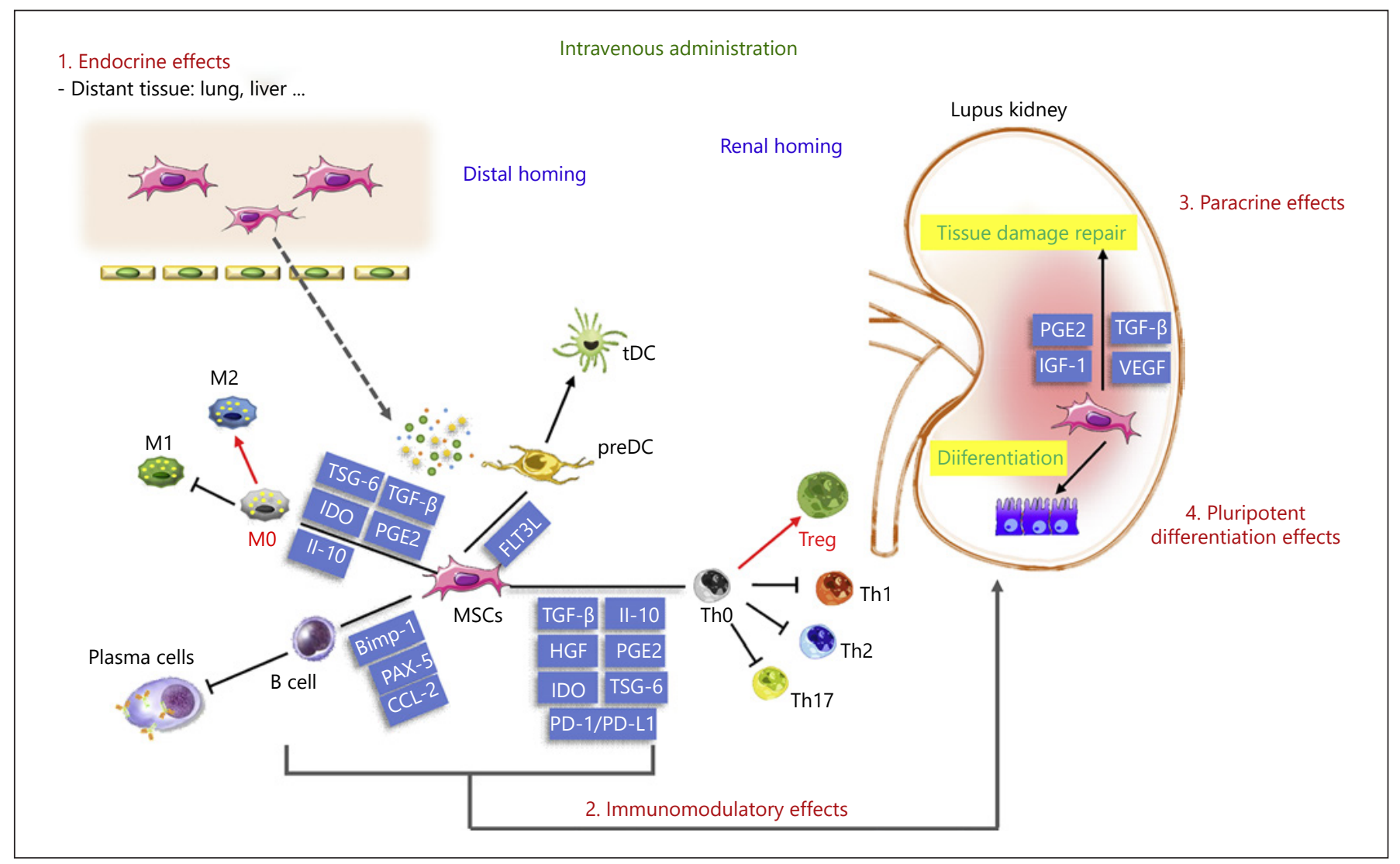

Fig. 2. The mechanism of allogeneic MSC therapy for LN: immunoregulatory function by secreting various soluble mediators and interacting with various immune cells such as T cells, B cells, macrophages, and DCs; promotion of repair and regeneration after homing to the kidney. MSC, mesenchymal stem cell; LN, lupus nephritis; tDC, tolerogenic dendritic cells.

tion, they specifically migrated to the sites of inflammation, and these exogenously infused MSCs responded to the injury signals by the corresponding cognate surface receptors [47]. Several cell-trafficking-related molecules have been demonstrated to be involved in this inflammation-directed homing process: adhesion molecules, chemokines, and matrix metalloproteinases. Among these chemokines, the chemokine ( $\mathrm{C}-\mathrm{X}$-C motif) ligand 12-chemokine (C-X-C motif) receptor 4 (CXCR4) and chemokine (C-C motif) ligand 2 (CXCL2)-chemokine (C-C motif) receptor 2 (CCR2) axes were most studied [48]. It is worth noting that all the homing-related molecules can be upregulated by inflammatory cytokines, such as TNF and IL-1. Thus, MSC engraftment and therapeutic efficiencies might depend on the different inflammation status (i.e., different levels of inflammatory cytokines).

In mice, it has been reported that ligation of CD 44 and hyaluronic acid mediated migration of MSCs to the injured kidney, and hyaluronic acid promoted MSC migra- tion in a dose-dependent manner. Moreover, renal homing of intravenously injected MSCs was blocked by preincubation with the CD 44 blocking antibody or by soluble hyaluronic acid [49]. Liu and colleagues found that, when administered systemically, MSCs homed to the ischemic kidney, improving renal function, accelerating mitogenic response, and reducing cell apoptosis, but these effects were abolished by either CXCR4 or CXCR7 inhibition, implicating the stromal derived factor-1-CXCR4/CXCR7 axis in kidney repair. Indeed, preconditioning MSCs with the mitogenic and prosurvival factor insulin-like growth factor (IGF)-1 before systemic infusion upregulated the expression of CXCR4 and restored renal function [50]. These findings may offer new insights into the mechanism involved in glomerular remodeling. Although it has been suggested that only $7-8 \%$ of mesangial cells were replaced by bone marrow-derived cells [51], the potential risk to the development of glomerular disease such as glomerulosclerosis could not be excluded. The differentiat- 
ing process and potential influence of MSC-derived mesangial cells in LN still need to be further studied. Several studies suggested that after engraft of the damaged renal tissue, MSCs integrated into tubular cells and differentiated into mesangial cells [52]. In swine renovascular disease, 4 weeks after intrarenal infusion, MSCs were detected in all regions of the kidney, but mostly at the renal interstation [53]. Our study observed that carboxyfluorescein diacetate succinimidyl ester-labeled MSCs could migrate to the kidney, with the highest detection level occurring at $24 \mathrm{~h}$ and sustaining for 1 week. Interestingly, the transfused MSCs could be detected in the kidney 11 weeks later. Further studies showed that these cells may regulate local inflammation by inhibiting monocyte chemotactic protein-1 (MCP-1) and high-mobility group box 1 [33] or repairing tissue damage by differentiating into functional renal tubular epithelial cells.

\section{Immunoregulatory Activities of MSCs}

The immunoregulatory activities of MSCs were through both cell-cell direct contact and the secretome, which was composed of soluble factors and extracellular vesicles [54, 55]. The soluble factors included NO, IDO, PGE2, tumor necrosis factor-inducible gene 6 protein (TSG6), CCL2, and PD-L1, IL-10, and TGF- $\beta 1$ [56-58]. Accumulated data suggested that MSCs exerted a profound influence on both innate and adaptive immune cells.

As for innate immune cells, MSCs played a key role in modulating the maturation and function of DCs [59], the potent antigen presenting cells. MSCs altered cytokine secretion from $\mathrm{DC} 1$ and $\mathrm{DC} 2$, stimulating a decrease in the secretion of TNF- $\alpha$ by DC1 and an increase in IL-10 from DC2 [60]. Moreover, our study showed that the number of peripheral tolerogenic $\mathrm{CD} 1 \mathrm{c}^{+} \mathrm{DCs}$ significantly decreased in SLE patients, especially those with LN. Transplantation of UC-MSCs dramatically upregulated CD1c ${ }^{+}$DCs. We found that UC-MSCs secreted FLT3L that binds to FLT3 on $\mathrm{CD} 1 \mathrm{c}^{+} \mathrm{DC}$ s to promote the proliferationandinhibit theapoptosisoftolerogenic $\mathrm{CD} 1 \mathrm{c}^{+} \mathrm{DCs}$. IFN- $\gamma$ induces FLT3L expression in UC-MSCs through the JAK/STAT signaling pathway. Thus, MSCs suppress inflammation in lupus through upregulating tolerogenic DCs [61]. For macrophages, MSCs have been shown to mediate the polarization of macrophages by skewing macrophages toward the M2 lineage, an anti-inflammatory phenotype $[62,63]$.

For adaptive immune cells, MSCs regulate T-cell function via 2 pathways. First, they could directly inhibit the functions of antigen-specific T cells. Second, they promoted the expansion of Treg cells and IL-10 expressing regulatory
B cells, which then suppressed the overactivated T cells [64]. MSCs inhibited the G1/S transition of the abnormal lupus $\mathrm{T}$ lymphocytes through upregulating p21 and p27 and downregulating cyclin-dependent kinase 2 [65]. Moreover, it has been demonstrated by Akiyama et al. [66] that BMMSCs induced the T-cell apoptosis via the FAS ligand-dependent FAS pathway, resulting in disease phenotype ameliorates. MSCs could secrete MCP-1, recruit T cells, and mediate T-cell apoptosis. The apoptotic $\mathrm{T}$ cells then induced macrophages to produce high levels of TGF- $\beta$, which upregulates Treg cells [66]. On the other side, Davies et al. [67] found that MSCs secreted PD-L1 and PD-L2, 2 ligands of the PD-1 receptor, to inhibit activation and proliferation of $\mathrm{T}$ cells, suppress $\mathrm{T}$-cell effector function, and modulate peripheral tolerance. Our group then unveiled that high levels of IFN $-\gamma$, produced predominantly by CD8 T cells in SLE patients, were a key factor involved in the stimulation of allogeneic UC-MSCs to produce IDO, which could then inhibit the proliferation of T cells from SLE patients [68]. Autoantibodies are predominantly produced with the help of Tfh cells and then form immune complexes that trigger LN. MSCs suppressed proliferation and differentiation of Tfh cells through iNOS (inducible NO synthase) production and a cell-contact-dependent manner $[36,69]$.

\section{Production of Trophic Factors}

Despite the crucial role attributed to MSC engraftment and immunoregulation at the site of injury, there is currently consensus that MSCs protect the damaged kidney also by the release of growth factors and proangiogenic factor. It has been demonstrated that cultured MSCs release large amounts of vascular endothelial growth factor and facilitate glomerular and tubular recovery [70]. In the model of chronic kidney disease, a single intravenous infusion of autologous MSCs triggered a significant increase in a group of nephrogenic proteins and transcription factors related to endothelial (the angiopoietin-1 receptor Tie-2) and epithelial (bone morphogenetic protein-7, Pax-2, and basic fibroblast growth factor) differentiation, in association with a marked improvement of renal function [71]. MSCs also produced IGF-1, and silence expression of IGF-1 limited the protective effect of MSCs on renal function and tubular structure in $\mathrm{mu}-$ rine cisplatin-induced kidney injury [51]. Previous studies have shown that pretreated MSCs with growth factors or gene modifications could enhance the therapeutic efficacy in wound-healing processes [72]. Thus, further understanding of the molecular pathways involved in the growth factor production will be of great help to develop better strategies for MSC-based therapies. 


\section{Conclusion}

The results of the first clinical trials with MSC therapy in severe SLE are undoubtedly encouraging. However, the efficacy of MSCs in the LN treatment remains confirmed. There are many confounding factors among the patients in the studies, including various disease severity, different complications, and the treating regimen. Moreover, the heterogeneity of MSCs as defined today and the intricate complication of cellular and humoral factors point to many questions to be resolved. Thus, future advances from stem cell science can be expected to pinpoint significant MSC subpopulations and/or specific stem-cell markers for immunoregulatory properties, as well as specific mechanisms of action, leading the way to the use of more potent stimulated or primed pretreated MSCs to treat LN.

\section{Conflict of Interest Statement}

The authors report no conflicts of interest in this work.

\section{Funding Sources}

The present work was supported by the Key Program of $\mathrm{Na}$ tional Natural Science Foundation of China (81930043), the Major International (Regional) Joint Research Project of China (81720108020), the National Natural Science Foundation of China (NSFC) (81771677), and Jiangsu Province Major Research and Development Program (BE2015602).

\section{Author Contributions}

L.W.C. and C.W.W. wrote the original manuscript. S.L.Y. organized sections of the manuscript. L.W.C. and C.W.W. prepared the figures. S.L.Y. revised the manuscript.

\section{References}

1 Murphy G, Isenberg DA. New therapies for systemic lupus erythematosus: past imperfect, future tense. Nat Rev Rheumatol. 2019 Jul;15(7):403-12.

2 Tsokos GC. Autoimmunity and organ damage in systemic lupus erythematosus. Nat Immunol. 2020 May;21(6):605-14.

3 Marion TN, Postlethwaite AE. Chance, genetics, and the heterogeneity of disease and pathogenesis in systemic lupus erythematosus. Semin Immunopathol. 2014 Sep;36(5): 495-517.

4 Anders H-J, Saxena R, Zhao M-H, Parodis I, Salmon JE, Mohan C. Lupus nephritis. Nat Rev Dis Primers. 2020 Jan;6(1):7.

5 Parikh SV, Almaani S, Brodsky S, Rovin BH. Update on lupus nephritis: core curriculum 2020. Am J Kidney Dis. 2020 Aug;76(2):26581.

6 Zhao J, Bai W, Zhu P, Zhang X, Liu S, Wu L, et al. Chinese SLE Treatment and Research Group (CSTAR) registry VII: prevalence and clinical significance of serositis in Chinese patients with systemic lupus erythematosus. Lupus. 2016 May;25(6):652-7.

7 Cheng RJ, Xiong AJ, Li YH, Pan SY, Zhang QP, Zhao Y, et al. Mesenchymal stem cells: allogeneic MSC may be immunosuppressive but autologous MSC are dysfunctional in lupus patients. Front Cell Dev Biol. 2019 Nov;7: 285.

8 An Y, Zhang H, Liu Z. Individualizing therapy in lupus nephritis. Kidney Int Rep. 2019 Oct;4(10):1366-72.

9 Appel GB, Contreras G, Dooley MA, Ginzler EM, Isenberg D, Jayne D, et al. Mycophenolate mofetil versus cyclophosphamide for induction treatment of lupus nephritis. J Am Soc Nephrol. 2009 May;20(5):1103-12.
10 Croca SC, Rodrigues T, Isenberg DA. Assessment of a lupus nephritis cohort over a 30year period. Rheumatology. 2011 Aug;50(8): 1424-30.

11 Sattwika PD, Mustafa R, Paramaiswari A, Herningtyas EH. Stem cells for lupus nephritis: a concise review of current knowledge. Lupus. 2018 Oct;27(12):1881-97.

12 Cervera R, Mosca M, Ríos-Garcés R, Espinosa G, Trujillo H, Bada T, et al. Treatment for refractory lupus nephritis: rituximab vs triple target therapy. Autoimmun Rev. 2019 Dec; 18(12): 102406.

13 Wang D, Niu L, Feng X, Yuan X, Zhao S, Zhang $\mathrm{H}$, et al. Long-term safety of umbilical cord mesenchymal stem cells transplantation for systemic lupus erythematosus: a 6-year follow-up study. Clin Exp Med. 2017 Aug; 17(3):333-40.

14 Chen Y, Yu Q, Hu Y, Shi Y. Current research and use of mesenchymal stem cells in the therapy of autoimmune diseases. Curr Stem Cell Res Ther. 2019;14(7):579-82.

15 Dominici M, Le Blanc K, Mueller I, SlaperCortenbach I, Marini F, Krause D, et al. Minimal criteria for defining multipotent mesenchymal stromal cells. The International Society for Cellular Therapy position statement. Cytotherapy. 2006;8(4):315-7.

16 Tang Y, Ma X, Zhang H, Gu Z, Hou Y, Gilkeson GS, et al. Gene expression profile reveals abnormalities of multiple signaling pathways in mesenchymal stem cell derived from patients with systemic lupus erythematosus. Clin Dev Immunol. 2012 Aug;2012:826182.

17 Fathollahi A, Gabalou NB, Aslani S. Mesenchymal stem cell transplantation in systemic lupus erythematous, a mesenchymal stem cell disorder. Lupus. 2018 Jun;27(7):1053-64.
18 Li X, Liu L, Meng D, Wang D, Zhang J, Shi D, et al. Enhanced apoptosis and senescence of bone-marrow-derived mesenchymal stem cells in patients with systemic lupus erythematosus. Stem Cells Dev. 2012 Sep;21(13): 2387-94.

19 Gao L, Bird AK, Meednu N, Dauenhauer K, Liesveld J, Anolik J, et al. Bone marrow-derived mesenchymal stem cells from patients with systemic lupus erythematosus have a senescence-associated secretory phenotype mediated by a mitochondrial antiviral signaling protein-interferon- $\beta$ feedback loop. Arthritis Rheumatol. 2017 Aug;69(8):1623-35.

$20 \mathrm{Ma}$ Y, Hendershot LM. ER chaperone functions during normal and stress conditions. J Chem Neuroanat. 2004 Sep;28(1-2):51-65.

21 Gu Z, Meng Y, Tao T, Guo G, Tan W, Xia $\mathrm{Y}$, et al. Endoplasmic reticulum stress participates in the progress of senescence of bone marrow-derived mesenchymal stem cells in patients with systemic lupus erythematosus. Cell Tissue Res. 2015 Aug;361(2): 497-508.

22 Gu Z, Tan W, Feng G, Meng Y, Shen B, Liu $\mathrm{H}$, et al. Wnt $/ \beta$-catenin signaling mediates the senescence of bone marrow-mesenchymal stem cells from systemic lupus erythematosus patients through the p53/p21 pathway. Mol Cell Biochem. 2014 Feb;387(1-2): 27-37.

23 Chen H, Shi B, Feng X, Kong W, Chen W, Geng L, et al. Leptin and neutrophil-activating peptide 2 promote mesenchymal stem cell senescence through activation of the phosphatidylinositol 3-kinase/Akt pathway in patients with systemic lupus erythematosus. Arthritis Rheumatol. 2015 Sep;67(9): 2383-93. 
24 Tan W, Gu Z, Shen B, Jiang J, Meng Y, Da Z, et al. PTEN/Akt-p27(kip1) signaling promote the BM-MSCs senescence and apoptosis in SLE patients. J Cell Biochem. 2015 Aug; 116(8):1583-94.

25 Ji J, Wu Y, Meng Y, Zhang L, Feng G, Xia Y, et al. JAK-STAT signaling mediates the senescence of bone marrow-mesenchymal stem cells from systemic lupus erythematosus patients. Acta Biochim Biophys Sin. 2017 Mar; 49(3):208-15.

26 Vazquez C, Horner SM. MAVS coordination of antiviral innate immunity. J Virol. $2015 \mathrm{Jul}$; 89(14):6974-7.

27 Moiseeva O, Mallette FA, Mukhopadhyay UK, Moores A, Ferbeyre G. DNA damage signaling and p53-dependent senescence after prolonged beta-interferon stimulation. Mol Biol Cell. 2006 Apr;17(4):1583-92.

28 Gu Z, Tan W, Ji J, Feng G, Meng Y, Da Z, et al. Rapamycin reverses the senescent phenotype and improves immunoregulation of mesenchymal stem cells from MRL/lpr mice and systemic lupus erythematosus patients through inhibition of the mTOR signaling pathway. Aging. 2016 May; 8(5): 1102-14.

29 Tang Y, Xie H, Chen J, Geng L, Chen H, Li X, et al. Activated NF- $\kappa B$ in bone marrow mesenchymal stem cells from systemic lupus erythematosus patients inhibits osteogenic differentiation through downregulating Smad signaling. Stem Cells Dev. 2013;22(4):66878.

30 Zhu Y, Feng X. Genetic contribution to mesenchymal stem cell dysfunction in systemic lupus erythematosus. Stem Cell Res Ther. 2018 May;9(1):149.

31 Sang A, Yin Y, Zheng YY, Morel L. Animal models of molecular pathology systemic lupus erythematosus. Prog Mol Biol Transl Sci. 2012;105:321-70.

32 Sun L, Akiyama K, Zhang H, Yamaza T, Hou Y, Zhao S, et al. Mesenchymal stem cell transplantation reverses multiorgan dysfunction in systemic lupus erythematosus mice and humans. Stem Cells. 2009 Jun;27(6):1421-32.

$33 \mathrm{Gu}$ Z, Akiyama K, Ma X, Zhang H, Feng X, Yao G, et al. Transplantation of umbilical cord mesenchymal stem cells alleviates lupus nephritis in MRL/lpr mice. Lupus. 2010 Nov; 19(13):1502-14.

34 Chang JW, Hung SP, Wu HH, Wu WM, Yang $\mathrm{AH}$, Tsai HL, et al. Therapeutic effects of umbilical cord blood-derived mesenchymal stem cell transplantation in experimental lupus nephritis. Cell Transplant. 2011;20(2):245-57.

35 Choi EW, Shin IS, Park SY, Park JH, Kim JS, Yoon EJ, et al. Reversal of serologic, immunologic, and histologic dysfunction in mice with systemic lupus erythematosus by long-term serial adipose tissue-derived mesenchymal stem cell transplantation. Arthritis Rheum. 2012 Jan;64(1):243-53.

36 Jang E, Jeong M, Kim S, Jang K, Kang BK, Lee DY, et al. Infusion of human bone marrowderived mesenchymal stem cells alleviates au- toimmune nephritis in a lupus model by suppressing follicular helper T-cell development. Cell Transplant. 2016;25(1):1-15.

37 Lazarus HM, Haynesworth SE, Gerson SL, Rosenthal NS, Caplan AI. Ex vivo expansion and subsequent infusion of human bone marrow-derived stromal progenitor cells (mesenchymal progenitor cells): implications for therapeutic use. Bone Marrow Transplant. 1995 Oct;16(4):557-64.

38 Newell LF, Deans RJ, Maziarz RT. Adult adherent stromal cells in the management of graft-versus-host disease. Expert Opin Biol Ther. 2014 Feb;14(2):231-46.

39 Sun L, Wang D, Liang J, Zhang H, Feng X, Wang $\mathrm{H}$, et al. Umbilical cord mesenchymal stem cell transplantation in severe and refractory systemic lupus erythematosus. Arthritis Rheum. 2010 Aug;62(8):2467-75.

40 Liang J, Zhang H, Hua B, Wang H, Lu L, Shi $S$, et al. Allogenic mesenchymal stem cells transplantation in refractory systemic lupus erythematosus: a pilot clinical study. Ann Rheum Dis. 2010 Aug;69(8):1423-9.

41 Gu F, Wang D, Zhang H, Feng X, Gilkeson GS, Shi S, et al. Allogeneic mesenchymal stem cell transplantation for lupus nephritis patients refractory to conventional therapy. Clin Rheumatol. 2014 Nov;33(11):1611-9.

42 Wang D, Zhang H, Liang J, Li X, Feng X, Wang $\mathrm{H}$, et al. Allogeneic mesenchymal stem cell transplantation in severe and refractory systemic lupus erythematosus: 4 years of experience. Cell Transplant. 2013;22(12):226777.

43 Deng D, Zhang P, Guo Y, Lim TO. A randomised double-blind, placebo-controlled trial of allogeneic umbilical cord-derived mesenchymal stem cell for lupus nephritis. Ann Rheum Dis. 2017;76(8):1436-9.

44 Wang D, Li J, Zhang Y, Zhang M, Chen J, Li $\mathrm{X}$, et al. Umbilical cord mesenchymal stem cell transplantation in active and refractory systemic lupus erythematosus: a multicenter clinical study. Arthritis Res Ther. 2014;16(2): R79.

45 Yang GX, Pan LP, Zhou QY, Song W, Chen ZQ, Wang CX, et al. Therapeutic effects of umbilical cord mesenchymal stem cells transplantation on systemic lupus erythematosus. Sichuan Da Xue Xue Bao Yi Xue Ban. 2014; 45(2):338-50

46 Van Pham P, Truong NC, Le PT, Tran TD, Vu $\mathrm{NB}$, Bui $\mathrm{KH}$, et al. Isolation and proliferation of umbilical cord tissue derived mesenchymal stem cells for clinical applications. Cell Tissue Bank. 2016 Jun;17(2):289-302.

47 Ullah M, Liu DD, Thakor AS. Mesenchymal stromal cell homing: mechanisms and strategies for improvement. iScience. 2019 May; 15: 421-38.

48 Karp JM, Leng Teo GS. Mesenchymal stem cell homing: the devil is in the details. Cell Stem Cell. 2009 Mar;4(3):206-16.

49 Herrera MB, Bussolati B, Bruno S, Morando L, Mauriello-Romanazzi G, Sanavio F, et al. Exogenous mesenchymal stem cells localize to the kidney by means of CD44 following acute tubular injury. Kidney Int. 2007 Aug; 72(4):430-41.

50 Liu H, Liu S, Li Y, Wang X, Xue W, Ge G, et al The role of SDF-1-CXCR4/CXCR7 axis in the therapeutic effects of hypoxia-preconditioned mesenchymal stem cells for renal ischemia/reperfusion injury. PLoS One. 2012;7:e4608.

51 Ito T, Suzuki A, Imai E, Okabe M, Hori M. Bone marrow is a reservoir of repopulating mesangial cells during glomerular remodeling. J Am Soc Nephrol. 2001 Dec;12(12): 2625-35.

52 Eirin A, Lerman LO. Mesenchymal stem cell treatment for chronic renal failure. Stem Cell Res Ther. 2014 Jul;5(4):83.

53 Eirin A, Zhu XY, Krier JD, Tang H, Jordan KL, Grande JP, et al. Adipose tissue-derived mesenchymal stem cells improve revascularization outcomes to restore renal function in swine atherosclerotic renal artery stenosis. Stem Cells. 2012 May;30(5):1030-41.

54 Heldring N, Mäger I, Wood MJ, Le Blanc K, Andaloussi SE. Therapeutic potential of multipotent mesenchymal stromal cells and their extracellular vesicles. Hum Gene Ther. 2015 Aug;26(8):506-17.

55 Phinney DG, Di Giuseppe M, Njah J, Sala E, Shiva S, St Croix CM, et al. Mesenchymal stem cells use extracellular vesicles to outsource mitophagy and shuttle microRNAs. Nat Commun. 2015 Oct; 6:8472.

56 Uccelli A, Moretta L, Pistoia V. Mesenchymal stem cells in health and disease. Nat Rev Immunol. 2008 Sep;8(9):726-36.

57 Keating A. Mesenchymal stromal cells: new directions. Cell Stem Cell. 2012 Jun;10(6): 709-16.

58 Bernardo ME, Fibbe WE. Mesenchymal stromal cells: sensors and switchers of inflammation. Cell Stem Cell. 2013 Oct;13(4):392-402.

59 Zhang W, Ge W, Li C, You S, Liao L, Han Q, et al. Effects of mesenchymal stem cells on differentiation, maturation, and function of human monocyte-derived dendritic cells. Stem Cells Dev. 2004 Jun;13(3):263-71.

60 Aggarwal S, Pittenger MF. Human mesenchymal stem cells modulate allogeneic immune cell responses. Blood. 2005 Feb;105(4):181522.

61 Yuan X, Qin X, Wang D, Zhang Z, Tang X, Gao X, et al. Mesenchymal stem cell therapy induces FLT3L and CD1c+ dendritic cells in systemic lupus erythematosus patients. Nat Commun. 2019 Jun;10(1):2498.

62 Selleri S, Bifsha P, Civini S, Pacelli C, Dieng MM, Lemieux W, et al. Human mesenchymal stromal cell-secreted lactate induces M2macrophage differentiation by metabolic reprogramming. Oncotarget. 2016 May;7(21): 30193-210.

63 de Witte SFH, Luk F, Sierra Parraga JM, Gargesha M, Merino A, Korevaar SS, et al. Immunomodulation by therapeutic mesenchymal stromal cells (MSC) is triggered through phagocytosis of MSC by monocytic cells. Stem Cells. 2018 Apr;36(4):602-15. 
64 Park MJ, Kwok SK, Lee SH, Kim EK, Park SH, Cho ML. Adipose tissue-derived mesenchymal stem cells induce expansion of interleukin-10-producing regulatory $B$ cells and ameliorate autoimmunity in a murine model of systemic lupus erythematosus. Cell Transplant. 2015;24(11):2367-77.

65 Ji S, Guo Q, Han Y, Tan G, Luo Y, Zeng F. Mesenchymal stem cell transplantation inhibits abnormal activation of Akt/GSK3 $\beta$ signaling pathway in $\mathrm{T}$ cells from systemic lupus erythematosus mice. Cell Physiol Biochem. 2012;29(5-6):705-12.

66 Akiyama K, Chen C, Wang D, Xu X, Qu C, Yamaza T, et al. Mesenchymal-stem-cell-induced immunoregulation involves FAS-ligand-/FAS-mediated T cell apoptosis. Cell Stem Cell. 2012 May;10(5):544-55.
67 Davies LC, Heldring N, Kadri N, Le Blanc K. Mesenchymal stromal cell secretion of programmed death-1 ligands regulates $\mathrm{T}$ cell mediated immunosuppression. Stem Cells. 2017 Mar;35(3):766-76.

68 Wang D, Feng X, Lu L, Konkel JE, Zhang H, Chen $\mathrm{Z}$, et al. A CD8 $\mathrm{T}$ cell/indoleamine 2,3-dioxygenase axis is required for mesenchymal stem cell suppression of human systemic lupus erythematosus. Arthritis Rheumatol. 2014 Aug;66(8):2234-45.

69 Zhang Z, Feng R, Niu L, Huang S, Deng W, Shi B, et al. Human umbilical cord mesenchymal stem cells inhibit $T$ follicular helper cell expansion through the activation of iNOS in lupus-prone B6.MRL-Fas(lpr) mice. Cell Transplant. 2017 Jun;26(6):1031-42.
70 Peired AJ, Sisti A, Romagnani P. Mesenchymal stem cell-based therapy for kidney disease: a review of clinical evidence. Stem Cells Int. 2016;2016:4798639.

71 Carrion FA, Figueroa FE. Mesenchymal stem cells for the treatment of systemic lupus erythematosus: Is the cure for connective tissue diseases within connective tissue? Stem Cell Res Ther. 2011 May;2(3):23.

72 Hahn JY, Cho HJ, Kang HJ, Kim TS, Kim MH, Chung JH, et al. Pre-treatment of mesenchymal stem cells with a combination of growth factors enhances gap junction formation, $\mathrm{cy}$ toprotective effect on cardiomyocytes, and therapeutic efficacy for myocardial infarction. J Am Coll Cardiol. 2008 Mar;51(9):933-43.

73 Ikehara S, Inaba M, Yasumizu R, Nagata N, Toki J, Hisha H, et al. Autoimmune diseases as stem cell disorders. Tohoku J Exp Med. 1994;173(1):141-155. 